\title{
Evolución del derecho a la salud en el ordenamiento jurídico Colombiano.
}

\section{Evolution of the right to health in the Colombian legal system}

\author{
Autores: Irma Raquel Ahumada Vargas ${ }^{1}$ \\ Cesar Noriel Muñoz Lozano² \\ Correspondencia: irmahumada@hotmail.com, cesarn.munoz@hotmail.com
}

\section{RESUMEN}

En el presente artículo de investigación se pondrá de relieve que el Estado Social de Derecho se fundamenta en la dignidad humana, por lo que la aplicación de los derechos fundamentales es una forma de garantizar al ser humano su bienestar, el de la familia y la comunidad, por ello mediante el presente artículo de reflexión se muestra el tratamiento jurídico que se le ha dado al derecho a la salud, pasando conceptualmente con el transcurrir tiempo como derecho de carácter prestacional, luego su reconocimiento por conexidad con otro derecho fundamental hasta convertir el derecho a la salud en un derecho fundamental autónomo mediante Ley Estatutaria 1751 de 2015.

\section{Palabras claves:}

Derecho a la salud, dignidad humana, derecho por conexidad, prestación social, derecho fundamental autónomo

\section{ABSTRACT}

In this research article will say the Social State of Law is based on human dignity, so the application of fundamental rights is a way of guaranteeing the human being's well-being, that of the family and the community, so through this article of reflection is shown The legal treatment that has been given to the right to health, passing conceptually with the passing of time as a benefit entitlement, then its recognition as a connection with another fundamental right to convert the right to health into an autonomous fundamental right through Statutory Law 1751 of 2015

\section{Key Words:}

Right to health, human dignity, right for connectedness, social provision, autonomous fundamental right.

\footnotetext{
${ }^{1}$ Irma Raquel Ahumada Vargas: Abogada Corporación Universitaria Americana, Contador Público, Universidad del Atlántico. Especialista en Derecho Constitucional de la Corporación Universitaria Americana.

${ }^{2}$ Cesar Noriel Muñoz Lozano: Abogado, Corporación Universitaria Americana, Contador Público, Universidad del Atlántico. Especialista en Derecho Constitucional de la Corporación Universitaria Americana.
} 


\section{Introducción}

Reiteradamente ha sido discutida la naturaleza jurídica del derecho a la salud para efectos de su reconocimiento, debido a que la importancia de su aplicación va más allá de una estricta prestación del servicio, es una fuente esencial de la vida que incorpora una serie de factores que integran la dignidad humana, la cual es el pilar y columna vertebral del Estado Social de Derecho Colombiano.

Este artículo tiene como propósito analizar el tratamiento y evolución del Derecho a la salud en el ámbito jurídico colombiano. Su alcance de aplicación es la normatividad legal del Derecho a la salud a partir de la expedición de la Constitución Política de 1991, y las normas internacionales que hacen parte del bloque constitucional. Está conformado por tres partes: en la primera se realiza una revisión de las bases constitucionales que garantizan y protegen al derecho a la salud; en la segunda se analiza la normatividad legal direccionada al reconocimiento y cumplimiento del derecho a la salud consagrado en la Constitución. Por último, se observa el protagonismo de la acción de tutela para el reconocimiento del derecho a la salud en Colombia

Finalizado el estudio, se expondrán las reflexiones del tema en cuanto a cuál ha sido el desarrollo jurídico del Derecho a la Salud en Colombia a partir de la Constitución de 1991 basados en los enfoques jurídicos, sus connotaciones sociales y las normas jurídicas que garantizan la materialidad del Derecho a la Salud. 


\section{Metodología}

Este trabajo ha sido realizado bajo la modalidad de investigación reflexiva con un enfoque de teoría analítica en el que el derecho a la salud se describe e interpreta bajo la óptica del paradigma iustnaturalista, que consagra la legitimidad del derecho positivo en concordancia con el derecho natural. Además es de corte descriptivo, con la aplicación del método deductivo.

\section{Resultados y discusiones.}

\section{Revisión de las bases constitucionales que garantizan y protegen el Derecho fundamental a la salud.}

La Constitución Política de 1991 dio lugar a la creación de mecanismos jurídicos que buscan garantizar la protección de los derechos fundamentales pero estos se han convertido en los medios requeridos, casi que obligatorios, para que los ciudadanos ejerzan sus derechos. Esta situación es una constante para el reconocimiento y exigibilidad del Derecho a la salud cuyo fundamento ha sido objeto de diversos debates dando como resultado una conceptualización que ha variado según el enfoque aplicado para su análisis desde la conversión de Colombia en un Estado Social de Derecho.

\subsection{El Derecho a la salud en el plano Constitucional Colombiano}

A pesar de la ratificación en 1936 del Pacto de Derechos Económicos, Sociales y Culturales, la salud solo empieza a ser reconocida como un derecho a partir de la Constitución de 1991 que señala como fin del Estado, 
garantizar la efectividad de los principios, derechos y deberes consagrados en su artículo 2, en el marco del Estado Social de Derecho. El artículo 44 consagra la salud como derecho fundamental de los niños y se fortalece con lo indicado en el artículo 50 de proteger a los menores de un año aunque no estén cubiertos por algún tipo de protección o de seguridad social; en el artículo 46 se indica que la protección y asistencia de las personas de la tercera edad; el artículo 48 establece que "la seguridad social es un servicio público de carácter obligatorio que se prestará bajo la dirección, coordinación y control del Estado"; en el artículo 49 se instaura que "la atención de la salud y el saneamiento ambiental son servicios públicos a cargo del Estado". (Constitución Política de Colombia, 1991)

Los servicios de promoción, protección y recuperación de la salud están en armonía con los artículos 365 y 366 que establecen que los servicios públicos son inherentes a la finalidad social del Estado y que es deber de éste asegurar su prestación eficiente a todos los habitantes del territorio nacional, de conformidad con la ley. (Constitución Política de Colombia, 1991).

Dentro de la constitución se encuentran artículos que tienen relación con el derecho a la salud y al estudiarse jurisprudencialmente han permitido resaltar su importancia, convirtiéndose en soporte esencial para el otorgamiento del derecho a la salud. Entre estos artículos encontramos: el artículo 11 que protege el derecho a la vida como derecho fundamental de mayor utilización dentro de las acciones de tutela por su directa conexidad; artículo 13 defiende el derecho a la igualdad pero en su inciso 3 hace énfasis en la protección que el Estado debe proveerle a "las personas que por su condición económica, física o mental, se encuentren en circunstancias de debilidad manifiesta" (Constitución Política de Colombia, 1991). 
La Corte Constitucional se ha pronunciado en múltiples ocasiones sobre la protección del derecho a la salud, en especial al efectuar revisiones vía de tutela para que los gobernados reciban una adecuada prestación del servicio. Además ha tocado, a través de jurisprudencia, otros aspectos del derecho a la salud incluyendo temas de control de riesgos sociales, el respeto al cuerpo y conceptos de salud asociados a comunidades étnicas y culturales, entre otros.

\subsection{El Derecho a la Salud y su relación con las Normas Internacionales que hacen parte del bloque de constitucionalidad}

Existe una serie de instrumentos jurídicos internacionales en los que está reconocido el derecho a la salud, en ellos están delimitadas la responsabilidad del Estado y las líneas de accesibilidad de los gobernados. Se hace mención, sin profundizar los conceptos, a los que hacen parte del la Organización de Naciones Unidas y los del Sistema Interamericano de Derechos Humanos. (Gómez Pavajeau, Maya Villazón, Linares Prieto, \& Uprimny, 2008)

\section{Sistema de la Organización de las Naciones Unidas}

- La Declaración Universal de Derechos Humanos protege explícitamente el derecho a la salud al decir que: "toda persona tiene derecho a un nivel de vida adecuado que le asegure, así como a su familia, la salud y en especial la alimentación, el vestido, la vivienda, la asistencia médica y los servicios sociales necesarios". (DDHH)

- El Pacto Internacional de Derechos Civiles y Políticos, en el artículo 7, dispone que "nadie será sometido a torturas ni a penas o tratos crueles, inhumanos o degradantes. En particular, nadie será sometido sin su libre consentimiento a experimentos médicos científicos". Este pacto fue aprobado por Colombia mediante la Ley 74 de 1968. (Naciones Unidas. Pacto Internacional de Derechos Civiles y Políticos, 1966) 
- El Pacto Internacional de Derechos Económicos Sociales y Culturales que entró en vigor para Colombia desde 1968, en su artículo 12 contiene una de las disposiciones de desarrollo más amplio en derecho internacional de los derechos humanos sobre el derecho a la salud. La posibilidad de cumplir el nivel de satisfacción del derecho estipulado en el Pacto, de ampliarlo a todos los sujetos sin discriminación y dentro de todos los elementos que lo constituyen, abarca la conformación de un sistema de protección que incluye derechos complementarios protegidos en otros instrumentos internacionales. En cuanto a las obligaciones surgidas del Pacto, la Observación General № 3, emitida por el mismo Comité, habla del compromiso de adoptar medidas pertinentes, no limitadas ni condicionadas por otras consideraciones, por ejemplo, de recursos. Al respecto el Comité pone de relieve que aunque se demuestre la insuficiencia de recursos disponibles, se mantiene la obligación de garantía y en consecuencia también la obligación de vigilar la realización o no realización de los derechos permanece. De manera análoga, subraya el Comité, aun en caso de limitaciones graves (crisis económica, procesos de ajuste u otros factores) se puede y se debe proteger a los grupos más vulnerables. (Gómez Pavajeau, Maya Villazón, Linares Prieto, \& Uprimny, 2008).

- La Convención Internacional sobre la Eliminación de Todas las Formas de Discriminación Racial (en vigor a partir de su aprobación mediante la Ley 22 de 1981) reconoce el derecho a la salud pública, la asistencia médica, la seguridad social y los servicios sociales. (Naciones Unidas Convención Internacional sobre la eliminación de todas las formas de discriminacion Racial)

- La Convención sobre la Eliminación de Todas las Formas de Discriminación contra la Mujer, (1979, para Colombia a partir de su aprobación mediante la Ley 1 de1981) incluye el derecho a la protección de la salud y a la seguridad en las condiciones de trabajo, incluso la salvaguardia de la función de reproducción; el acceso a los servicios que se refieren a la 
planificación familiar, el período posterior al parto, y la nutrición adecuada durante el embarazo y lactancia (Naciones Unidas.Convencion Internacional, 1979)

- La Convención sobre los Derechos del Niño (1989, en vigor para Colombia desde su aprobación a través de la Ley 12 de 1991) demanda en el artículo 24 la plena aplicación del derecho a la salud y la adopción de medidas mediante, entre otras cosas, la aplicación de la tecnología disponible, el suministro de alimentos nutritivos adecuados y agua potable salubre; la atención sanitaria prenatal y posnatal apropiada a las madres; y el desarrollo de atención sanitaria preventiva. (Naciones Unidas. Convencion sobre Derechos del Niño, 1989)

\section{Sistema Interamericano de Derechos Humanos}

- La Declaración Americana de los Derechos y Deberes del Hombre consagra el derecho de toda persona a la preservación de la salud y el bienestar.

- La Convención Americana de Derechos Humanos afirma en su artículo 26 que los Estados partes se comprometen "a adoptar providencias, tanto a nivel interno como mediante la cooperación internacional, especialmente económica y técnica" para lograr progresivamente la plena efectividad de los derechos contenidos en la Carta de la Organización de los Estados Americanos, que en su artículo 33 hace referencia al derecho a la salud. (Organizacion de los Estados Americanos, 1948).

- Protocolo Adicional a la Convención Americana sobre Derechos Humanos en materia de Derechos Económicos, Sociales y Culturales, o Protocolo de San Salvador suscrito en 1988, aprobado por Colombia en 1996 y que entró en vigor en 1999, protege igualmente el derecho a la salud en su Artículo 10. (Organizacion de los Estados Americano, 1969) 
A partir de las normas internacionales y los desarrollos que sobre estas han producido los órganos autorizados, se ha consolidado una doctrina sobre los contenidos del derecho y las obligaciones estatales para la materialización de los mismos distinguiéndose usualmente cuatro componentes normativos, que en el caso del derecho a la salud se caracterizan de la siguiente manera:

- Disponibilidad. Consiste esencialmente en que exista oferta suficiente de servicios e infraestructura asociados al goce del derecho a la salud, que se traduce en contar con un número suficiente de establecimientos, bienes y servicios de salud.

- Accesibilidad. Consiste en que las personas no tengan obstáculos para acceder al derecho, lo que se expresa en el acceso efectivo a los establecimientos, bienes y servicios de salud sin discriminación alguna, en condiciones que permitan el acceso físico, el económico y que puedan acceder a la información.

- Aceptabilidad. Está vinculada no sólo a la calidad que deben tener las prestaciones suministradas sino a la conformidad de las condiciones de realización del derecho con el contexto y a su relación con otros derechos. La aceptabilidad también implica el respeto por "el derecho a controlar su salud y su cuerpo, con inclusión de la libertad sexual y genésica, y el derecho a no padecer injerencias, como el derecho a no ser sometido a torturas ni a tratamientos y experimentos médicos no consensuales".

- Calidad. Tienen que ver con el derecho a que la prestación de los servicios sea llevada a cabo por personal idóneo, capacitado y que disponga de condiciones laborales adecuadas, que el servicio sea prestado en condiciones satisfactorias, que se brinden medicamentos de calidad y atención oportuna. (Gómez Pavajeau, Maya Villazón, Linares Prieto, \& Uprimny, 2008) 
2. Análisis de la normatividad legal direccionada al reconocimiento y cumplimiento del Derecho a la Salud consagrado en la Constitución.

Después de las nefastas consecuencias de la Segunda Guerra Mundial, la dignidad humana es comprendida como una condición propia de los seres humanos y está considerada como uno de los principales objetivos que deben proteger los Estados. De tal manera que así lo vienen manifestando las Constituciones proclamadas en la posguerra, entre ellas la Constitución de 1991. Antes de revisar de la normatividad legal colombiana es preciso resaltar la importancia de los artículos 48 y 49 de la Constitución Política por ser estos la base estructural del Sistema Integral de Seguridad Social.

\section{1‥ Breve análisis de los artículos 48 y 49 de la Constitución Política de Colombia}

Se considera que el artículo 48 es uno de los componentes más relevantes en materia de seguridad social, ya que garantiza este derecho irrenunciable a todos los integrantes del territorio Colombiano, especialmente en salud, con la debida intervención del Estado, los particulares y la comunidad en general, bajo la orientación de tres principios fundamentales: eficacia, universalidad y solidaridad. Estos principios son puntos esenciales de engranaje del sistema de Seguridad social y a su vez se constituyen en criterios para orientar, tanto al legislador como a los administradores de justicia, a una adecuada y correcta interpretación.

El artículo 49 "La atención de la salud y el saneamiento ambiental son servicios públicos a cargo del Estado. Se garantiza a todas las personas el acceso a los servicios de promoción, protección y recuperación de la salud". De esta manifestación se observan las siguientes características: 
- Establece la responsabilidad directa del Estado en garantizar la prestación de los servicios en salud. Por lo que se puede inferir que a pesar de que sean particulares los que lleven a cabo la actividad, el Estado es el garante para que esto sea cumplido adecuadamente.

- La universalidad es un principio que se aprecia en la expresión "garantizar a todas las personas" debido a que no es a unas sí y a otras no, a quienes se les da la atención sino que es para todos los habitantes del territorio colombiano.

- El acceso al servicio de salud es incondicional ya que su otorgamiento se da sin discriminación en todas las etapas de la vida. A su vez esta situación da el carácter de irrenunciable puesto que está inmerso por la naturaleza en el ser humano.

- La integralidad se refiere a la cobertura del sistema, es decir, al propósito de responder a situaciones de contingencia que afecten o pongan en riesgo la salud. Esto incluye su capacidad económica y de todas aquellas condiciones que se requieran con el fin de asegurar la vida de la comunidad.

Es importante mencionar que el legislador en aras de cumplir con la obligación de disminuir las necesidades básicas de la población más necesitada apostó desarrollar estos mandatos constitucionales mediante la expedición de la ley 100 de 1993. Estableciendo dos sistemas generales de atención a la seguridad social." (Ley 100, 1993)

El primero, destinado a atender el sistema de seguridad social en pensión, y el segundo a brindar la atención de la seguridad social en salud contenido en Libro II Capitulo Primero. Este último es el marco de estudio de la presente investigación, y comprende dos regímenes bien definidos:

“a) Contributivo $y$, 
b) Subsidiado, basados en un criterio predominante, más no único, como lo es la capacidad de pago. En efecto, en el régimen contributivo la persona afiliada cuenta con capacidad de pago y por ello se le exige el pago de una cotización o aporte; en el régimen subsidiado la persona afiliada carece de esa capacidad de pago, por pertenecer a la franja de población que por su condición económica y social corresponde a la más pobre y vulnerable del país y, por consiguiente, no está obligada a realizar dichos aportes”. (Sentencia C-130, 2002)

En este mismo sentido el Derecho a la "seguridad social está dada por el conjunto estructurado de normas principios y técnicas cuyo objetivo es atender la satisfacción de las necesidades sociales derivadas de la realización de determinadas contingencias que se estiman socialmente dignas de protección. El instrumento que da vida al derecho por medio de expedición de normas, y nace condicionado a la eficacia que dispone el estado, especialmente de tipo económica y financiera con que pueda dotarlo, tales condiciones imponen la delimitación de las necesidades sociales a cubrir, y contribuyen a separar la política del derecho de seguridad social, el cual debe establecerse en cada sistema jurídico (Torregrosa Sanchez, 2009, pág. 68 y 69)

La seguridad social aparece entrelazada con las políticas del estado y se concibe como: la protección del hombre contra todas las necesidades sociales mediante la toma de una serie de medidas tendientes a la satisfacción de las personas a través del suministro de medios que les permitan solucionar los problemas de subsistentencia.

Se aprecia que en los artículos 48 y 49 la intención del Constituyente es que este sistema sea incluyente y beneficioso a toda la población sin que tenga que mediar un vínculo contractual o laboral. El objetivo principal es brindar cobertura a toda la población por lo que se hace necesario que exista un 
manejo adecuado de recursos, sufragado por la misma población en la medida de las capacidades económicas de cada uno de sus miembros.

La titularidad del derecho a la salud es irrenunciable, ya que este es necesario para que el ser humano desarrolle todas sus capacidades de vida bajo el tan promulgado concepto de dignidad humana. Para su efectiva materialización, desde los primeros años de aplicación de la Carta Magna, las Altas Cortes manifestaron su posición frente a la naturaleza jurídica del derecho a la salud tal como lo expresó la Corte Constitucional:

"El derecho a la salud conforma, en su naturaleza jurídica, un conjunto de elementos que pueden agruparse en dos grandes bloques: el primero, que lo identifica como un predicado inmediato del derecho a la vida, de manera que atentar contra la salud de las personas equivale a atentar contra su propia vida. Por estos aspectos, el derecho a la salud resulta un derecho fundamental. El segundo bloque de elementos, sitúa el derecho a la salud con un carácter asistencial, ubicado en las referencias funcionales del denominado Estado Social de Derecho, en razón de que su reconocimiento impone acciones concretas. La frontera entre el derecho a la salud como fundamental y como asistencial es imprecisa y sobre todo cambiante, según las circunstancias de cada caso, pero en principio, puede afirmarse que el derecho a la salud es fundamental cuando está relacionado con la protección a la vida. Los derechos fundamentales, solo conservan esta naturaleza, en su manifestación primaria, y pueden ser objeto allí del control de tutela. Como es necesario proteger el derecho a la salud del actor, no cabe duda de que él puede reclamarlo de cualquier institución de asistencia pública, donde se presten tales servicios, en forma gratuita, en virtud del deber general del Estado de garantizar la salud de este tipo de enfermos" (Sentencia T-484, 1992). 


\section{1 b. Principales normas legales del derecho a la salud desde la perspectiva de una prestación del servicio.}

Al analizar el carácter asistencial del derecho a la salud es necesario referenciar el ordenamiento jurídico establecido por el Estado para ejercer esta función. La implementación con una estructura organizada fue establecida por primera vez en 1975 como Sistema Nacional de Salud y fue definido como "..conjunto de organismos, instituciones, agencias y entidades que tengan como finalidad específica procurar la salud de la comunidad en los aspectos de promoción, protección, recuperación y rehabilitación" (Decreto 056. Art. 1, 1975).

Quince años después se expidió la Ley 10 de 1990 para reorganizar el Sistema Nacional de Salud para mejorar la cobertura y calidad de los servicios prestados a la comunidad. Contempló, entre otros aspectos: la financiación, administración, gestión, control, accesibilidad e infraestructura del Sector salud de tal modo que estuvieran en armonía la normatividad internacional, la política de descentralización administrativa y fiscal y las políticas gubernamentales. Es de resaltar que esta ley fue básica para la organización de la prestación de servicios de la salud al considerarlo como un servicio público a cargo de la nación, tal como lo declara en su artículo $1^{\circ}$ (Ley 10, 1990).

Frente al Estado Social de Derecho de la nueva Constitución Política de 1991 se toma el concepto de seguridad social cuya pretensión es el amparo de los individuos, a través de medidas orientadas a proporcionarles seguridad durante toda su vida, e incluso después de fallecido, de manera que pueda desarrollar su existencia, su personalidad y su vejez sin tener que preocuparse de llegar a la indigencia. Este criterio constituye el desarrollo de los lineamientos del artículo 48 de la Carta Superior (Torregrosa 
Sanchez, 2009). En atención a lo anterior se promulga la Ley 100 de 1993 que en su artículo $1^{\circ}$ define el Sistema de Seguridad Social Integral:

"El sistema de seguridad social integral tiene por objeto garantizar los derechos irrenunciables de la persona y la comunidad para obtener la calidad de vida acorde con la dignidad humana, mediante la protección de las contingencias que la afecten. El sistema comprende las obligaciones del Estado y la sociedad, las instituciones y los recursos destinados a garantizar la cobertura de las prestaciones de carácter económico, de salud y servicios complementarios, materia de esta Ley, u otras que se incorporen normativamente en el futuro" (Ley 100, 1993).

La Ley 100/93 introdujo un nuevo esquema estructural a la salud en las que destacan como características principales la promoción de la utilización adecuada de los recursos mediante la asignación de funciones a organismos de control, la vinculación del sector privado para la prestación del Servicio de salud, la libre escogencia de las Entidades prestadoras del servicio por parte de los usuarios, y la ampliación de la cobertura de acuerdo a los estándares universales, entre otras.

La Ley 1122 de 2007 hizo modificaciones al Sistema General de Seguridad Social en Salud (SGSSS), se cambiaron los aspectos de dirección, universalización, financiación, equilibrio entre los actores del sistema, racionalización, y mejoramiento en la prestación de servicios de salud, fortalecimiento en los programas de salud pública y de las funciones de inspección, vigilancia y control y la organización y funcionamiento de redes para la prestación de servicios de salud (Cárdenas Ramirez, 2013).

En el 2011 fue expedida la Ley 1438 en donde destaca la prestación gratuita de los servicios y medicamente a niños y adolescentes y la obligación de informar a las entidades competentes los casos en que se detecten indicios de maltrato físico, psicológico o violencia sexual (Ley 1438, 2011). 
En procura de mejorar el Sistema de Salud toda estas Leyes han tenido modificaciones, además de la expedición de Decretos, Resoluciones y Acuerdos por parte de los Entes Reguladores de la salud en Colombia.

\subsection{Reconocimiento condicionado del Derecho a la salud.}

El derecho a la salud por regla general tiene reconocimiento condicionado bajo dos aspectos especiales. El primero de ellos se origina a partir del individuo que tiene una cualidad especial y está amparado expresamente por la Constitución. El segundo aspecto o condición depende de la relación o conexidad existente entre el derecho a la salud con otro derecho fundamental, por lo general el derecho a la vida.

\subsubsection{Protección de Individuos con protección especial}

El derecho a la salud has sido tratado jurisprudencialmente como un derecho fundamental autónomo para casos de personas que tienen protección especial. Entre ellos: los menores de edad, adultos mayores, los discapacitados y todos aquellos que se encuentren en estado de debilidad manifiesta.

Cuando se trata de menores, la Corte se ha manifestado a su favor en la mayoría de los casos, aun cuando no se de total cumplimiento a determinados requisitos legales. Como ejemplos tenemos: (Sentencia T286 , 1998) señala en qué casos procede la inaplicabilidad de las disposiciones legales o reglamentarias para el acceso a tratamiento y medicamentos excluidos del Plan Obligatorio en Salud; (Sentencia T-945, 2006) incluye el principio Pro-Homine que consiste en aplicar la interpretación más favorable para proteger los derechos de las personas; (Sentencia T-165, 1995) y (Sentencia T-628, 1998) indican que se debe dar la atención al menor aunque no cumpla con las semanas mínimas de cotización que están reglamentadas en el Decreto 806 de 1998. 
La Corte ha considerado que debe existir el compromiso del Estado para proteger a la madre gestante no solo por la función de perpetuar la especie humana sino por su dignidad como persona y la prevalencia del cuidado de los niños. Por lo tanto se han emitido sentencias en protección al embarazo como: (Sentencia T-765, 2000) que dice: "Frente al cambio normativo en cuanto a la exigencia de los requisitos para adquirir el derecho al reconocimiento de las prestaciones económicas derivadas de la licencia de maternidad, debe aplicarse la norma más favorable a la trabajadora.". De igual manera se protege el acceso a recibir tratamiento y medicamentos durante el embarazo, así como solicitar el pago a la licencia de maternidad con el fin de resguardar a la madre y al hijo que acaba de nacer, quienes se convierten en personas en estado de debilidad manifiesta y como tal poseen amparo especial (Sentencias: T-928A/02, T-996/2002, T350/2007).

En cuanto a los adultos mayores, de manera general la Corte se ha pronunciado por asuntos más que de salud son de tipo laboral relacionadas con el acceso al Sistema Pensional. La corte, incorpora el concepto de integralidad para paciente en estado de avanzada edad, o ancianos (Sentencia T-001, 2000)

\subsubsection{Derecho a la salud en conexidad con otros derechos fundamentales}

Desde la constitución de 1886, el derecho a la salud, se manejaba como una prestación de servicio en su Artículo 19 (Cosntitución Politica Colombiana, 1886), tampoco quedo definida de manera clara en la Constitución de 1991, y solo la Corte Constitucional en sus pronunciamiento judiciales, después de un minucioso estudio, utilizando para ellos, métodos de interpretación sistemático e integral y axiológica de la Constitución Política, propende por 
considerar que el derecho a la salud es un derecho fundamental, en tres dimensiones:

a) Derecho a la salud por conexidad con la vida "opera en conexión con el derecho a la vida, luego si hay peligro de que ésta sea afectada cabe la acción de tutela. Con este punto de apoyo garantista la jurisprudencia de la Corte Constitucional protege muchos casos en relación a la prestación del servicio de salud." (Sentencia SU-562, 1999).

b) Derecho a la salud por integralidad de las personas," implica la prestación oportuna, continua e ininterrumpida por parte de los prestadores de asistencia en salud y la entrega de los medicamentos, insumos y servicios que se requieran para la recuperación de la salud. " (Sentencia T- 736, 2016)

c) Derecho a la salud por dignidad humana, es uno de los derechos tipificados como fundamentales innominados, por la corte constitucional, consagrado en el artículo 94 de la Constitución Política, cuyo titularidad recae solamente a las personas, así mismo la corte ha planteado, que el derecho a la dignidad tiene como fin proteger a las personas en los siguientes aspectos: La autonomía individual, condiciones materiales para el logro de una vida digna y la integridad física y moral que resulte necesaria para lograr la inclusión social de una persona excluida 0 marginada (Rodriguez, 2014)

La Corte Constitucional manifestó: "en la revisión de los fallos de tutela proferidos por todos los Jueces de la República para amparar los derechos fundamentales de los individuos, ha creado líneas jurisprudenciales que protegen el derecho a la salud, visto ya no desde su relación de conexidad con el derecho a la vida, el derecho a la integridad personal y el derecho a la dignidad humana, ni como derecho fundamental en contextos donde el tutelante es un sujeto de especial protección, sino como derecho fundamental autónomo, que enmarca el "estado completo de bienestar físico, mental y social" que le permiten al individuo desarrollar las diferentes 
actividades propias de los seres humanos, y que propenden por su dignificación." (Sentencia T-920, 2011).

En este sentido la corte ha jalonado como órgano de control constitucional de los derechos a la salud, a darle esa categoría de Derecho fundamental autónomo que más adelante se abordará con las precisiones jurídicas contempladas en la reciente ley estatutaria de la reforma a la salud.

\subsection{Derecho a la salud como un Derecho fundamental autónomo. Ley 1751 de 2015.}

La Ley Estatutaria 1751 de 2015, surge con la finalidad, no sólo de proteger el derecho a la salud, sino con el objetivo de ser revisor de su garantía, como lo expresa su artículo 2:

“... El derecho fundamental a la salud es autónomo e irrenunciable en lo individual y en lo colectivo. ... Comprende el acceso a los servicios de salud de manera oportuna, eficaz y con calidad para la preservación, el mejoramiento y la promoción de la salud. El Estado adoptará políticas para asegurar la igualdad de trato y oportunidades en el acceso a las actividades de promoción, prevención, diagnóstico, tratamiento, rehabilitación y paliación para todas las personas. De conformidad con el artículo 49 de la Constitución Política, su prestación como servicio público esencial obligatorio, se ejecuta bajo la indelegable dirección, supervisión, organización, regulación, coordinación y control del Estado".

El sistema de salud es definido en el artículo 4 y estipula las herramientas de los que debe disponer el Estado para materializar el derecho fundamental a la salud y cumplir con las obligaciones asignadas en el artículo 5. En el artículo 6, la ley estatutaria se estipulan cuatro elementos fundamentales 
para la garantía del derecho fundamental a la salud: disponibilidad, aceptabilidad, accesibilidad e idoneidad y calidad profesional.

En los artículos 7, 8 y 9 se instaura la obligación del Estado de realizar evaluaciones anuales sobre los resultados de la efectiva materialización del derecho a la salud de manera integral por parte de los agentes del sistema de salud. La información obtenida será tomada como base para la adopción de políticas públicas que promuevan el mejoramiento continuo de la salud.

De manera especial, el capítulo II en sus artículos del 12 al 16, se contemplan la garantía y mecanismos de protección que procuran la participación de parte de los usuarios en las decisiones de los agentes del sistema de salud, la eliminación de barreras que impidan el acceso a los servicios de salud y los procedimientos para solucionar controversias presentadas entre los actores del sistema y se pueda llegar a un acuerdo entre las partes afectadas según cada caso en particular (Ley Estatutaria 1751., 2015).

El tercer capítulo hace referencia a la autonomía profesional, se propende por el respeto a la dignidad de los profesionales y trabajadores de la salud

\section{Protagonismo de la acción de Tutela para el reconocimiento del derecho a la salud en Colombia.}

La acción de tutela es uno de los mecanismos e instrumentos del sistema jurídico colombiano que ha influido en la constitucionalización del derecho, en temas relacionados, tales como el derecho de petición, especialmente en el derecho a la salud y otros establecidos en la Constitución y que han permitido limitar la acciones de poder de los funcionarios públicos a favor de los conciudadanos. 
Su origen en gran medida obedeció a unas series de pacto internacionales en el cual el Estado Colombiano entro a hacer partes de los pactos, previa aprobación dada por el Congreso de la Republica, entre ellos se encuentran los siguientes:

a) Pacto Internacional de derechos civiles y políticos, que prevé en su artículo $2^{\circ}$ numeral 3, cada persona cuyos derechos a libertades reconocidas en el presente pacto hayan sido violados podrá interponer un recurso efectivo, aun cuando tal violación hubiera sido cometida por personas que actuaban en ejercicios de sus funciones oficiales.

b) Convención de derechos humanos numeral 1 del artículo 25, que estableció una protección judicial en los siguientes términos: toda persona tiene derecho a un recurso sencillo y rápido o a cualquier otro recurso efectivo antes los jueces o tribunales competentes, que la ampare contra actos que violen sus derechos fundamentales reconocidos por la Constitución, en la ley o la presente Convención, aun cuando tal violación sea cometida por personas que actúen en ejercicio de sus funciones públicas.

En este orden de ideas, la acción de tutela el Constituyente la incorpora en la Constitución de 1991, articulo 86,quedando definida en los siguientes términos; "toda persona y en cualquier momento podrá reclamar ante los jueces a través de un procedimiento preferente y sumario, ya sea de manera personal, o por quien actué en su nombre, la protección inmediata de sus derechos constitucionales fundamentales, cuando quiera que estos resulten vulnerado, y procederá cuando la persona afectada no disponga de otro medio de defensa judicial, con el fin de evitar un perjuicio irremediable".

La acción de tutela, en materia normativa ha tenido un desarrollado, ajustados a los contenidos de normas expedidas de carácter reglamentarias procesales, (Decreto ley 2591 por el cual se reglamenta la accion de tutela, 1991), Decreto 3061992 y Decreto 1382 del 2000, por su parte a nivel 
jurisprudencial, ha sido bastante riguroso en sus aspecto formal y de fondo, especialmente las relacionadas con los derechos a la salud, desde el año 1992 hasta julio de 2017 se han interpuesto más de siete millones de tutelas, (Periodico Nuevo Siglo, 2016) destacándose en su mayor participación de la comunidad, los derecho de peticiones y en segundo orden los relacionados con reclamaciones por afectaciones del derecho a la salud, por ser este ultimo en que está dirigido el análisis, se muestras las siguientes Tablas:

Tabla No 1. Tutelas presentadas en materia de salud

\begin{tabular}{|c|c|c|c|c|}
\hline Año & $\begin{array}{l}\text { Población } \\
\text { Afiliada }\end{array}$ & $\begin{array}{l}\text { Tutelas } \\
\text { Presentadas }\end{array}$ & $\begin{array}{l}\text { Aumento o } \\
\text { Disminucion }\end{array}$ & $\begin{array}{l}\text { Variacion } \\
\%\end{array}$ \\
\hline 2000 & 23.017 .000 & 24.843 & & \\
\hline 2001 & 23.806 .139 & 34.310 & 9.467 & 27,59 \\
\hline 2002 & 24.194 .854 & 42.734 & 8.424 & 19,71 \\
\hline 2003 & 25.413 .805 & 51.944 & 9.210 & 17,73 \\
\hline 2004 & 30.040 .650 & 72.033 & 20.089 & 27,89 \\
\hline 2005 & 33.981 .988 & 81.017 & 8.984 & 11,09 \\
\hline 2006 & 36.461 .003 & 96.226 & 15.209 & 15,81 \\
\hline 2007 & 38.655 .698 & 107.238 & 11.012 & 10,27 \\
\hline 2008 & 39.561 .521 & 142.957 & 35.719 & 24,99 \\
\hline 2009 & 38.681 .796 & 100.490 & (42.467) & $-42,26$ \\
\hline 2010 & 40.047 .220 & 94.502 & $(5.988)$ & $-6,34$ \\
\hline 2011 & 41.999 .763 & 105.947 & 11.445 & 10,80 \\
\hline 2012 & 42.854 .962 & 114.313 & 8.366 & 7,32 \\
\hline 2013 & 42.879 .901 & 115.147 & 834 & 0,72 \\
\hline 2014 & 43.515 .870 & 117.743 & 2.596 & 2,20 \\
\hline
\end{tabular}

Total $\quad \mathbf{1 . 3 0 1 . 4 4 4}$

Fuente: Ministerio de Salud y protección Social

Fuente: Defensoría del Pueblo

Tabla $\mathbf{N}^{\circ} 2$ Sentencias Decantadas por tutelas

\begin{tabular}{|c|c|c|c|c|}
\hline Año & $\begin{array}{l}\text { Tutelas } \\
\text { Decantadas }\end{array}$ & $\begin{array}{c}\text { Tutelas } \\
\text { Decantadas } \\
\text { en } \\
\text { salud }\end{array}$ & $\begin{array}{l}\text { Aumento o } \\
\text { Disminucion }\end{array}$ & Variacion \\
\hline 1992 & 182 & 8 & & \\
\hline
\end{tabular}


Vol.9, No 1. Diciembre de 2017 pp. 1-32

\begin{tabular}{|c|c|c|c|c|}
\hline 1993 & 394 & 20 & 12 & 60,00 \\
\hline 1994 & 360 & 29 & 9 & 31,03 \\
\hline 1995 & 403 & 32 & 3 & 9,38 \\
\hline 1996 & 370 & 11 & -21 & $-190,91$ \\
\hline 1997 & 376 & 22 & 11 & 50,00 \\
\hline 1998 & 565 & 24 & 2 & 8,33 \\
\hline 1999 & 705 & 69 & 45 & 65,22 \\
\hline 2000 & 1.340 & 140 & 71 & 50,71 \\
\hline 2001 & 976 & 139 & -1 & $-0,72$ \\
\hline 2002 & 784 & 140 & 1 & 0,71 \\
\hline 2003 & 868 & 187 & 47 & 25,13 \\
\hline 2004 & 898 & 219 & 32 & 14,61 \\
\hline 2005 & 1.061 & 151 & -68 & $-45,03$ \\
\hline 2006 & 845 & 209 & 58 & 27,75 \\
\hline 2007 & 903 & 216 & 7 & 3,24 \\
\hline 2008 & 997 & 140 & -76 & $-54,29$ \\
\hline 2009 & 760 & 141 & 1 & 0,71 \\
\hline 2010 & 872 & 151 & 10 & 6,62 \\
\hline 2011 & 784 & 35 & -116 & $-331,43$ \\
\hline 2012 & 877 & 44 & 9 & 20,45 \\
\hline 2013 & 764 & 1 & -43 & $-4300,00$ \\
\hline 2014 & 789 & 26 & 25 & 96,15 \\
\hline 2015 & & & -1 & $-4,00$ \\
\hline
\end{tabular}




\begin{tabular}{|l|l|l|l|l|} 
& 615 & 25 & & \\
\hline 2016 & 536 & 40 & 15 & 37,50 \\
\hline 2017 & 216 & 21 & -19 & $-90,48$ \\
\hline
\end{tabular}

Total $\quad 0 \quad 2.240$

Fuente: Corte Constitucional Informe estadístico año 2017

Fuente: Propia. Se efectuó conteo de la página Web, Corte Constitucional.

De acuerdo a las variaciones presentadas en tabla $N^{\circ} 1$ con relación a los periodos anteriores, se observas que el numero de tutela correspondientes a los periodos 2000 al 2014 muestra un incrementos sucesivos de año en año, con excepción de los año 2009 y 2010, que muestran descenso en el número de tutelas presentadas, sin embargo los resultados denotan un constante incremento que obedecen en gran parte por el incumplimiento en la atención de los servicios médicos a sus afiliados, entre otros, todos aquellos relacionados con los derechos a la salud. Ejemplo, entrega de medicamentos, citas medicas prolongadas etc.

En la tabla $\mathrm{N}^{\circ} 2$, el resultado muestra las variaciones de las tutelas revisada por la corte Constitucional desde el año 1992 hasta junio de 2017.

\subsection{Seguimiento de tutelas revisadas por la Corte Constitucional.}

La corte ha permanecido abierta, evacuando un cúmulos de tutelas presentadas por los afiliados al sistemas de salud, en este orden se han emitidos más de dos mil doscientos cuarenta (2240) sentencias en materia de salud, en su mayoría abordando temas relacionadas al cumplimiento del mandato constitucional, de garantizar el derecho a la salud, y otras apuntan 
a realizar seguimiento de la recomendaciones impartidas por el mismo órgano jurisdiccional, con el fin de forjar a los órganos de Dirección y Administración, a establecer mecanismo jurídico que permita a las instituciones cumplir de manera material y efectiva los servicios de salud de la población colombiana, con fundamento a los principios de accesibilidad, eficacia, cobertura, y calidad. Como parámetro de análisis del presente seguimiento es revisar determinadas sentencias en sus aspectos jurídicos, que abordan el cumplimiento por parte de los entes encargadas de direccionar, las rutas de acción de los servicios de salud, las cuales se mencionan las siguientes:

- Sentencia T-527/93. La corte trata de equilibrar el sistema de salud partiendo de los recursos disponibles con la carga que le exige la atención de la población, haciendo uso racional de los mismos: "No existen los recursos para prestar un servicio eficiente a toda la población. Partiendo de la insuficiencia descrita, es evidente que los recursos disponibles deben utilizarse en los casos en que realmente sea posible recuperar la salud. No tiene sentido el ocupar cuartos o camas de hospital con personas a quienes se han prestado todos los servicios posibles, sin que exista la esperanza de una mejoría en su estado de salud, pues al hacerlo se priva a otro de la atención que en su caso sí podría tener un resultado aceptable. (Sentencia T-527, 1993)"

- Sentencia T-447/94. La corte incorpora un nuevo elemento, la prevalencia del derecho a la salud de los niños y que recoge postura jurídica de la normatividad internacional. "En lo que hace al derecho a la salud de los niños, al tenor del artículo 44 superior, éste no sólo es fundamental sino prevalente, en el sentido de su respeto incondicional y universal. Por ello el Estado tiene en el cumplimiento de un derecho uno de sus objetivos primordiales, razón por la cual goza de especial 
protección por el orden jurídico. La incondicionalidad de tal bien hace que el Estado de manera prevalente asegure, en la medida de sus posibilidades y capacidades, su eficacia. De ahí que lo tutele cuando se lesiona o amenace en forma grave, ya que contra el derecho a la salud de un niño no puede haber argumentación válida alguna." (Sentencia T447, 1994).

- Sentencia T-409/95. La Corte en este pronunciamiento ratifica lo postura de sentencias anteriores catalogado el derecho a la salud de naturaleza prestacional adicionando una nueva variable para tratarlo como fundamental al existir conexidad con la vida:

“El derecho a la salud, comprendido dentro del catálogo de los derechos sociales, económicos y culturales tiene en la Constitución un contenido evidentemente prestacional, pues al deber correlativo que tiene toda persona de "procurar el cuidado integral de su salud y la de su comunidad", se encuentra el del Estado de garantizar su cumplimiento, a través del correspondiente sistema de servicios, mediante el suministro de prestaciones concretas en materia de salud. Pero es de anotar que la cobertura y extensión del servicio de salud a los diferentes sectores de la comunidad y las condiciones y la eficiencia de su prestación, aun cuando son tareas prioritarias de la acción estatal, necesariamente dependen de las políticas globales y de desarrollo económico y social, las cuales se encuentran sujetas y limitadas a la disponibilidad de recursos. El derecho a la salud no fue consagrado en la Constitución, salvo con respecto a los niños como un derecho fundamental. No obstante, la Corte ha sido reiterativa en el sentido de considerarlo como un derecho fundamental por conexidad, cuando en casos concretos debidamente sopesados y analizados por el juez de tutela, la protección de la salud involucre al mismo tiempo el amparo de la vida misma." (Sentencia T-409, 1995) 
Con posterioridad las Sentencias T-121 de 2005 y T-724 de 2008 hacen parte de la línea jurisprudencial para que el derecho a la salud sea considerado como derecho fundamental autónomo bajo los criterios de conexidad con la vida.

- Sentencia SU-480/97. En el análisis de la corte de siete sentencias, emite pronunciamiento sobre una serie de enfoque relacionada, con la interpretación de la norma, con criterio de integrar la normatividad que regulan el derecho a la salud, y la seguridad social. para que sea acogida por los jueces en sus fallos. (Sentencia SU-480, 1997).

- Sentencia T-238/96. La Corte en esta parte sigue sosteniendo que el derecho a la salud es prestacional e igual que la seguridad social, pero señala unas condiciones en las cuales no es aplicable para las normas que rigen para el derecho a la salud: Falta de medicamentos por tratamiento excluido por la reglamentación legal o administrativa, en este se considera amenaza de los derechos constitucionales. Segundo, lugar de medicamentos que no puedan ser sustituido por uno de los contemplado en el plan obligatorio en salud, y que no tenga el mismo nivel del requerido para el tratamiento, y en tercer lugar que el paciente ralamente no pueda sufragar los costo, ni acceder por otro medio al sistema de salud:

"En general, se ha entendido que los derechos a la salud y a la seguridad social, en razón a que tienen un contenido prestacional y dependen de ciertas condiciones para su aplicación, las más de las veces condiciones económicas, pertenecen a una categoría distinta de los derechos constitucionales fundamentales, cuya aplicación no puede condicionarse en manera alguna $y$, por tal razón, el Constituyente los reguló en un capítulo distinto al de éstos, 
considerando aquéllos como derechos sociales, económicos y culturales. Así, a los colombianos se les garantiza el acceso a mecanismos que les permitan tener una salud y una seguridad social adecuadas, pero en manera alguna ellas mismas, pues la garantía efectiva de conseguir la salud, entendida como normal funcionamiento corporal, escapa a las posibilidades de un Estado. Por tales razones, la acción de tutela no procede directamente para el amparo de esos derechos, pues son derechos constitucionales, pero con un carácter distinto al de los fundamentales, para los cuales está reservado, en principio, el mecanismo judicial de protección a que se refiere el artículo 86 de la Carta. No obstante, esta Corporación ha sostenido que cuando la amenaza o vulneración de tales derechos implique, a su vez, una amenaza o vulneración de derechos constitucionales con rango fundamental, el juez constitucional está autorizado para protegerlos indirectamente a través de la tutela, aplicándola a derechos que, como aquellos a los cuales se ha hecho referencia, no son fundamentales." (Sentencia T-236, 1998)

- Sentencia SU-760/2008. Es una de las sentencia con mayor impacto en comunidad colombiana, pues hace el más alto contenido jurídico, marcando las pautas que debe seguir el sistema de salud. La corte aborda el estudio dentro del proceso de revisión de veinte y siete expedientes de tutelas presentados donde se invoca la protección de derechos fundamentales en salud, tales como acceso a los servicios de salud los cuales habían sido negado por las entidades prestadoras de servicios, acceso al servicio de salud no contemplado en el pos, acceso a los servicios de salud que requiere un menor para su adecuado desarrollo; reconocimiento de incapacidades laborales cuando no se cumplen los requisitos de pago oportuno; acceso a los 
servicios de salud en condiciones de integralidad; acceso a los servicios de salud de alto costo y para tratar enfermedades catastróficas, así como a los exámenes diagnósticos; acceso a los servicios de salud requeridos por personas vinculadas al Sistema de Salud, en especial si se trata de menores; acceso a los servicios de salud cuando se requiere desplazarse a vivir en lugar distinto a aquel en que reside la persona; libertad de elección de la 'entidad encargada de garantizarle el acceso a la prestación de los servicios de salud" y duda acerca de la inclusión del lente intraoculares etc. (Sentencia SU$760,2008)$.

La corte en conclusión allega en los siguientes términos: queda claro que 'la salud' no es un asunto estrictamente privado que pueda ser relegado a la suerte de cada individuo. La posibilidad de morir o sufrir graves daños a la integridad personal a causa de una epidemia o una enfermedad endémica, aun cuando se contara con recursos económicos suficientes para costearse buenos servicios de atención médica, llevó a que la salud se convirtiera en un asunto de gran importancia pública. (Sentencia SU-760, 2008)

\section{Conclusiones}

Como se ha observado durante el desarrollo de la investigación, a partir de la promulgación de la Constitución Política de 1991 el derecho a la salud ha tenido una evolución jurídica que tiene como base estándares internacionales que el Estado Colombiano desde sus inicios ha procurado cumplir de manera organizada, para ello expidió la Ley 100 de 1993 que contiene el Sistema de Seguridad Social en Salud. Posteriormente de 
acuerdo a las necesidades sociales se han promulgado un conjunto de normas jurídicas dirigidas a mejorar la calidad en la prestación del servicio en salud, lo que deja ver el carácter asistencial que tiene la legislación del Sistema de Seguridad en Salud en referencia al derecho a la salud.

Los cambios normativos del derecho a la salud obedecen esencialmente a la influencia de factores sociales y a los continuos pronunciamientos en sentencias de las Altas Cortes en respuestas a las numerosas tutelas presentadas para tener acceso a este derecho. Esta situación se ha convertido en una constante, ya que en ocasiones si no hay tutela no hay otorgamiento del servicio por parte de las Instituciones encargadas de la prestación del servicio. Para estos entes, en su mayoría privados, la salud es un negocio y como tal busca cumplir solo con lo básico exigido por el sistema para obtener mayor rendimiento económico.

Otros de los alcances que ha tenido el desarrollo del derecho a la salud en materia jurídica, son las reiteradas posturas de la Corte Constitucional dada en los fallos, en la cual para el reconocimiento de los derechos, ha abordas distintos enfoque de materialización del derecho a la salud de acuerdo al siguiente esquema:

- Por conexidad con el derecho a la vida u otros derechos fundamentales

- Por Integralidad, es decir en personas que se encuentran en estado de debilidad, o incapacidad física, mental que le impida disfrutar del bienestar requerido a una vida digna.

- Por Dignidad humana, criterio que emerge de la misma condición de ser persona, catalogado como derechos innominados, aunque no estén codificado en el texto normativo.

Estos criterios de la corte, acompañados de una serie de recomendaciones, dio impulso para que se produjeran cambios importantes y de gran 
significado en la normatividad jurídica y presupuestaria apuntando a buscar una estabilidad en aspectos financieros y cumplimientos de los derechos, en la operatividad del sistema de salud, que a través de la historia ha estado bajo la lupa del colapso, y fue así, que el Congreso de la Republica expidió la ley Estatutaria (1751 de 2015) que incorpora el derecho a la salud, como un Derecho Fundamental Autónomo.

En nuestro criterio el derecho a la salud, su recorrido a través del tiempo, siempre se ha caracterizado por tener simplemente acceso a los servicio de una atención médica, cuando en contrario esta debe ser más integral, preventivo e intervenido para gozar del más alto nivel de bienestar dable en salud, en cuanto a lo físico, mental y psíquico.

Es conveniente precisar que la titularidad del derecho a la salud es irrenunciable. Para materializarlo efectivamente, los esfuerzos del Estado deben ir más allá de la emisión de normas jurídicas sino que además se complementen con unos mecanismos de prevención, seguimiento y control con los cuales el Sistema de Seguridad Social funcione de manera integral y efectiva para que se convierta en una herramienta esencial para que el ser humano desarrolle su potencial de vida bajo el concepto de dignidad humana.

\section{Referencias}

Agudelo, S. J. \& Calderón, M. A. (2016). Responsabilidad patrimonial del Estado colombiano por actos de reforma constitucional. En Justicia, 29, 99-118. http://dx.doi.org/10.17081/just.21.29.1236

Cárdenas Ramirez, E. (8 de Mayo de 2013). Alcance del derecho a la salud en colombia: una revisión constitucional, legal y jurisprudencial. Revista de Derecho Universidad del Norte (40), 198-225. 
Constitución Política de Colombia. (Julio de 1991).

Cosntitución Politica Colombiana (1886).

Correa, M., Jaimes, J., Cardozo, S. \& Mantilla, C. (2013). Estrategia para la disminución de violencias en la escuela en la ciudad de Bucaramanga. Revista Justicia, 18(24), 14-24. Recuperado de http://publicaciones.unisimonbolivar.edu.co/rdigital/ojs/index.php/justicia/arti cle/viewFile/136/138

DDHH. (s.f.). Decalración Universal de Derechos Humanos adoptada y proclamada en Asamblea General. Resolucion 217A. Diciembre 10 de 1948.

Decreto 056. Art. 1 (Presidencia de la República. 15 de Enero de 1975).

Decreto ley 2591 por el cual se reglamenta la accion de tutela (Presidencia de la República 19 de 11 de 1991).

Gómez Pavajeau, C., Maya Villazón, E., Linares Prieto, P., \& Uprimny, R. (Mayo de 2008). El Derecho a la salud en perspectiva de Derechos Humanos y el Sistema de Inspección, Vigilancia y Control del Estado Colombiano en Materia de Quejas en Salud. Bogotá, Colombia.

Ley 10 (Congreso de Colombia 10 de Enero de 1990).

Ley 100. (1993). Congreso de la República.

Ley 1438, articulos 18 y 21 (Congreso de la República de Colombia 19 de Enero de 2011). 
Ley Estatutaria 1751. (16 de Febrero de 2015). .Congreso de la República.

Naciones Unidas Convención Internacional sobre la eliminación de todas las formas de discriminacion Racial. (s.f.). Adoptada por Resolución 2106A de Diciembre 21 de 196.

Naciones Unidas. Convencion sobre Derechos del Niño. (1989). Adoptada por Asamblea General Resolución 44/2 Noviembre 20.

Naciones Unidas. Pacto Internacional de Derechos Civiles y Políticos. (16 de Diciembre de 1966). Adoptado por Asamblea General en Resolucion 2200 A (III).

Naciones Unidas.Convencion Internacional. (18 de 12 de 1979). Asamblea General, Resolucion 34/180.Convención Internacional sobre toda forma de discriminación contra la mujer.

Organizacion de los Estados Americano. (22 de 11 de 1969). Convencion Americana sobre Derechos Humanos.

Organizacion de los Estados Americanos. (1948). Novena Conferencia Internacional Americana sobre derechos y Deberes del hombre. Bogota, Colombia.

Periodico Nuevo Siglo. (23 de 08 de 2016). Jueces han evacuado 723 mil tutelas año 2015. (htt//www.elnuevosiglo.com.co/index.php/articulos/07-2016, Ed.) Jueces han evacuado 723 mil tutelas año 2015(07). 
Rodriguez, C. A. (5 de 12 de 2014). Origen,Evolucion Jurisprudencial y Alternativas a su Interpretacion. Origen, Evolucion Jurisprudencial y Alternativas a su Interpretacion. Bogota, Colombia.

Sentencia C-130 (Corte Constitucional 2002).

Sentencia SU-480 (Corte Constitucional 25 de Septiembre de 1997).

Sentencia SU-562, Expedienete T-192384y T 193556 (Corte Constitucional 04 de 08 de 1999).

Sentencia SU-760 (Corte Constitucional 31 de Julio de 2008).

Sentencia T- 736, T-5752232 (Corte Costitucional 19 de 12 de 2016).

Sentencia T-001 (Corte Constitucional 12 de Enero de 2000).

Sentencia T-121 (Corte Contitucional 2015).

Sentencia T-165 (Corte Constitucional 19 de Abril de 1995).

Sentencia T-236 (Corte Constitucional 1998).

Sentencia T-286 (Corte Constitucional 4 de junio de 1998).

Sentencia T-409 (Corte Constitucional 12 de Septiembre de 1995). 
Sentencia T-447 (Corte Constitucional 13 de Octubre de 1994).

Sentencia T-484 (Corte Constitucional 11 de Agosto de 1992).

Sentencia T-527 (Corte constitucional 1993).

Sentencia T-628 (Corte Constitucional 4 de Noviembre de 1998).

Sentencia T-724, T 1857757 (Corte Constitucional 22 de 07 de 2008).

Sentencia T-765 (Corte Constitucional 22 de Junio de 2000).

Sentencia T-920 (Corte Constitucional 7 de Diciembre de 2011).

Sentencia T-945 (Corte Constitucional 16 de Noviembre de 2006).

Sentencias: T-928A/02, T-996/2002, T-350/2007 (Corte Constitucional).

Torregrosa Sanchez, A. E. (2009). Introducción al Derecho de la Seguridad Social. Bogotá: Grupo Editorial Ibañez.

Trejos, L. (2013). Aproximación a las dinámicas del crimen organizado en la ciudad de Barranquilla. El caso del Frente José Pablo Díaz de las Autodefensas Unidas de Colombia (200-2006). Justicia, 118-134. 
Vega, H. (2015). Aspectos dogmáticos y políticos criminales de la estructura general del delito en el sistema penal colombiano. Justicia, 27, 42-72. Recuperado de http://dx.doi.org/10.17081/just.3.27.320

Yáñez, M. (2013). El desplazamiento forzado en la jurisprudencia de la Corte Constitucional colombiana (1991-2003): momento previo a la declaratoria forma del estado de cosas inconstitucional. Revista Justicia, (23), 191-218. Barranquilla: Universidad Simón Bolívar 